\title{
How the UAE can reduce the prevalence of obesity among the youth?- a health policy perspective
}

\begin{abstract}
Nutritional health is a global concept and fosters much debate. The UAE youths' frequency of being more obese is twice to be thrice greater than the published international standards. The United Arab Emirates schoolchildren/youth (ages 1018) are said to be 1.8 times more obese than those in the United States, according to statistics from the First United States National Health and Nutrition Examination Survey. According to World Health Organisation report, the UAE has been classified as the second highest for obesity rates in the world. As a result of these obesity statistics among the youths, there are associated diseases, which tend to plague the UAE adults later including cardiovascular diseases, diabetes, hypertension and others. The Global School-based Student Health Survey highlighted on the increasing prevalence of obesity in UAE in terms of measuring the dietary behaviors and physical activity of students in grades 8 to 10 . The theoretical framework for this policy brief will be the Health Belief Model. Therefore, this policy brief highlights the public health issue within the UAE context is obesity among the youths, and the framework for implementing a public health campaign among the UAE youths, whereby, contributing to attaining one of the UAE Vision 2021 National agenda targets for reducing the prevalence of obesity.
\end{abstract}

Volume 6 Issue 2 - 2017

\author{
Immanuel Azaad Moonesar, Cynthia J Lewis \\ Hickman
}

Mohammed Bin Rashid School of Government, UAE

Correspondence: Immanuel Azaad Moonesar, Assistant Professor of Health Policy, Mohammed Bin Rashid School of Government Level 13, Convention Tower P.O. Box 72229, Dubai, UAE, Tel +97| 43175533 ,

Email immanuel.moonesar@mbrsg.ac.ae

Received: November 09, 2016 | Published: February 07, 2017

\section{UAE public health campaign introduction}

The United Arab Emirates (UAE) Vision 2021 National Agenda aims to achieve a world-class healthcare system. Within this National Agenda, one indicator is the prevalence of obesity among children ${ }^{1}$ which has been adopted from the World Health Organisation. In 2010, the prevalence of obesity among children was $14.4 \%$ in the UAE; such that this national agenda indicator targets to reduce the current value by $17 \% .{ }^{1}$ The UAE schoolchildren/youth (ages 10-18) are said to be 1.8 times more obese than those in the United States, according to statistics from the First United States National Health and Nutrition
Examination Survey (NHANES-1). ${ }^{2}$ The UAE youths' frequency of being more obese is twice to be thrice greater than the published international standards. ${ }^{3-6}$ United Arab Emirates (UAE) is part of the GCC countries and ranks 18 on 2007 WHO list of the fattest countries with $68.3 \%$ of its citizens with an unhealthy weight. ${ }^{7}$ In another study (2010), the Global School-based Student Health Survey (GSHS) on the United Arab Emirates measured the dietary behaviours and physical activity of students in grades 8,9 and 10, ages 13-15years. The Table 1 shows the results of students aged 13-15years pertaining to dietary behaviours and Table 2 demonstrates the results of students aged 13-15years pertaining to physical activity.

Table I Results shows UAE students aged I3-15 years pertaining to dietary behaviours

\begin{tabular}{|c|c|c|c|}
\hline Results for students aged I3-15years, 2010 & Total & Boys & Girls \\
\hline Percentage of students who were underweight ( $<-2 \mathrm{SD}$ from median for BMI for age and gender) & 4.I (3.0-5.5) & $5.5(3.6-8.3)$ & $3.1(2.1-4.6)$ \\
\hline Percentage of students who were overweight ( $>+$ ISD from median for BMI for age and gender) & $38.4(35.0-41.8)$ & $42(37.1-47.1)$ & $35.9(32.1-39.9)$ \\
\hline Percentage of students who were obese ( $>+2 \mathrm{SD}$ from median for BMl for age and gender) & $14.4(12.4-16.7)$ & $18.2(|5.2-2| .7)$ & II $.8(9.5-14.5)$ \\
\hline $\begin{array}{l}\text { Percentage of students who usually drank carbonated soft drinks one or more times per day } \\
\text { during the past } 30 \text { days }\end{array}$ & $42(38.2-45.9)$ & $50.3(45.0-55.5)$ & $36.5(32.7-40.5)$ \\
\hline
\end{tabular}

Source: Global School-based Student Health Survey United Arab Emirates http://www.who.int/chp/gshs/UAE_20I0_FS.pdf

Table 2 Results shows UAE students aged 13-15 years pertaining to physical activity

\begin{tabular}{|c|c|c|c|}
\hline Results for students aged I3-I 5years, 2010 & Total & Boys & Girls \\
\hline $\begin{array}{l}\text { Percentage of students who were physically active for a total of at least } 60 \text { minutes per day on } \\
\text { five or more days during the past seven days }\end{array}$ & $27.5(24.5-30.7)$ & $34.5(31.0-38.2)$ & $22.8(18.9-27.4)$ \\
\hline $\begin{array}{l}\text { Percentage of students who went to physical education (PE) class on three or more days each } \\
\text { week during the school year }\end{array}$ & $27.8(24.8-31.1)$ & $29.4(24.7-34.5)$ & $26.9(23.3-30.7)$ \\
\hline $\begin{array}{l}\text { Percentage of students who spent three or more hours per day during a typical or usual day } \\
\text { doing sitting activities }\end{array}$ & $51.2(47.0-55.3)$ & $44.8(39.7-50.0)$ & $55.3(50.2-60.4)$ \\
\hline
\end{tabular}


Furthermore, according to World Health Organisation (WHO) report in the global news, the UAE was classified as the second highest for obesity rates in the world. As a result of these obesity statistics among the youths, there are associated diseases, which tend to plague the UAE adults later, such cardiovascular diseases, diabetes, hypertension and others. ${ }^{2}$ The prevalence of obesity amongst children indicator measures the proportion of children between the age of 5 and 17 who are considered obese out of the total children of the same age group. ${ }^{1}$ Obesity in children is defined as follows: ${ }^{1}$

a. Children aged 5 to 12: The proportion of children with a BMI greater than 2 standard deviations above the growth standard median

b. Children aged above 12: The proportion of children with a BMI greater than 30 .

Such a UAE public health campaign intervention and strategies is urgently needed at the Federal level in order to identify overweight and obese youths. In 2000, researchers reported that obesity preventative health intervention was needed and urged UAE health officials of the potentially increased serious youth overweight/obesity related morbidity; ${ }^{1}$ furthermore, more recently researchers concur that there is more an urgent need for addressing this health concern among the youth. ${ }^{8}$ There is a need to aggressively asses these urgent areas of the UAE youth health status and formation effective preventative health interventions such as the HEAL public health campaign. Therefore, the public health issue within the UAE context is obesity among the youths.

\section{Healthy eating requires understanding nutritional health}

Nutritional health is a global concept. Regardless of the citizenry, nutrients to maintain proper and adequate nutrition are essential. It requires knowledge about the nutritional health habits practiced in your surroundings. Understanding the patterns that influences eating patterns, nutritional decision-making may become easier. One way to determine nutritional health patterns is to evaluate the consumer's grocery shopping experience and dining-out experience. Without an awareness of proper nutrition, the foods and amount of foods consumed can lead to obesity and other health related conditions. ${ }^{9}$

\section{UAE public health campaign need}

For this public health campaign, the Health Belief Model (HBM) is utilized and can be defined individual's perception of the risk of a health issue/problem and where the evaluation of commended behaviour(s) for precluding or dealing the problem are presented,,$^{10,11}$ The key concepts and factors of HBM which are addressed are perceived susceptibility, perceived severity, perceived benefits of action, cues to action and self-efficacy. ${ }^{11,12}$ The justifications for using the HBM in the public health campaign of promoting healthier nutrition eating habits and choices, entitled: "HEAL," that is, Healthy Eating and Active Living. It is paramount to be capable in attaining the perceived severity and its associated benefits, in terms of specifying obesity consequences and its course of actions and recommendation, in addition to the positive results and potential outcome in the long run. ${ }^{11}$ The HEAL public health campaign would be competent to be able to determine the perceived barriers, in terms of tailoring the health educational information to the best of accuracy; cues to action, in terms of presenting the factors involved for the readiness to change; and its self-efficacy elements, in terms of providing appropriate educational materials and nutritional counseling in performing action. ${ }^{11}$
The overall purpose of the HEAL public health campaign implementation and launch could be to make the youth more aware of healthier eating styles and active living exercises. Another goal could be to equip and provide the youth with the ways of expanding and improving their nutrition programs/diets through practice and exercises, which would bring about the social change among the youth and in turn assist in behavioral changes for a positive outcome.

Therefore, the target audience could be UAE youth with ages of 15 to 29 years. Initially, the writer thought of selecting the schoolchildren and youth age group. The main reason for selecting the UAE youth in universities/schools is that most of these youth are situated in the university residences, where they have a direct influence over their eating habits and food choices. Another reason is that 75 percent of youth both males and females have basic knowledge on health and diseases associated with eating habits. ${ }^{13}$ Furthermore, health awareness would be appropriate for this target audience in order to increase the health knowledge percentage.

This public health campaign could be conducted over a semester long as a general-education course at the first-year of any Bachelor's degree program at the universities/schools across the UAE. Such a public health campaign initiative has been prompted from the numerous reports from the local and world news, in addition to research publications. ${ }^{13-15}$

\section{Public health communication tools \& recommendations}

It is evident on the recent disaster of the Japan earthquake and tsunami that the uses of contemporary social media communication tools such as Face book, YouTube and Twitter were very effective and useful in the dissemination of information and highlights during that time.$^{16}$ As highlighted earlier, the public health issue within the UAE context is obesity among the youths. Among the youths in the Arab countries, social-networking sites (SNS) are very popular. ${ }^{17}$ On average, the Arab youth has 100 persons attached to their account. ${ }^{17} \mathrm{It}$ is known that among the Arab youths, the SNS plays an integral part of shaping the perception of the political views and Arab cultures and customs. $^{18}$

In a recent research report from the Mohammed Bin Rashid School of Government, there were over 400,000 new Facebook users. In the UAE, during the period of January to May 2014, $48 \%$ of these new Facebook users were within the age group of 15-29years. ${ }^{19}$ Furthermore, the UAE is the second highest in the Arab world to have a high penetration rate of $58 \%$, indicating persistent growth and pervasive usage of Facebook within the UAE society. ${ }^{20,21}$

Consequently, the use of Facebook, LinkedIn and YouTube would be best useful and effective for the Healthy Eating \& Active Living (HEAL) public health campaign at the universities/schools among the UAE. The use of internet videos and audios could be convenient to be utilized as resources for the campaign and can be posted onto Facebook and LinkedIn since all the potential participants in the campaign are highly active on this example of SNS. ${ }^{16}$ Furthermore, SNS has become a daily routine and activity among the Arab youths: ${ }^{16,22,23}$ therefore, the use of SNS such as Facebook and LinkedIn could be appropriate communication social media tools.

There are two ways for adjusting the HEAL public health campaign based on the type of social media to be used in the campaign. One way is to have the university youth to create HEAL discussion groups on distinct topics on Facebook and LinkedIn. All the youth 
within the particular HEAL campaign own at least a blackberry smart phone where both applications of Facebook and LinkedIn are presently functioning. Therefore, there could be 24-7 interactions and discussions on both applications on the blackberry smart phone. As the facilitator, the topics could allow for much engagement and interaction and would allow for the youth to post applicable videos and audios with respects to the HEAL public health campaign. Such a method has been proven to be functional and effective in the classroom setting among Arab youths. ${ }^{16}$ Another way is to link the public health message directly to their status updates (via the Facebook accounts and LinkedIn), since on average blackberry users have at least 50 blackberry contacts. Therefore, overall the reach of the audience is much wider and mainly among the Arab youths. ${ }^{16}$

There are three reasons for the adjustments of public health message as highlight above. It is indeed critical to understand that the public health message has to be tailored to target audience, taking into account, the age group, and their community and literacy levels. As identified earlier, 75 per cent of university youth both males and females have basic knowledge on health and diseases associated with eating habits. ${ }^{12}$ Therefore, the pitch of literacy levels would be at a university literacy level. Furthermore, among the Arab youths, there is much influence on their cultures, habits and customs based on their daily interactions on SNS. ${ }^{16}$ As a result, the main three reasons of adjustments would be to have an effective problem solution to promoting healthier lifestyle and daily habits; to allow reaching the target audience effectively which would indeed encourage for constructive interactions and discussions; and to empower these youths as the future social change agents in making healthier choices and encouraging other's peers to indulge in healthier living and make it as the 'new norm'.

There are two ways on marketing the HEAL public health campaign. The first would be to create an information packet on the new health awareness courses and/or workshops at the UAE universities/schools. This could allow for the providence of the course information, including statistics on the prevalence of obesity and its future consequences on the Arab youths ${ }^{1,2}$ to the first-year youth at universities / schools where there could be credits awarded for the successful completion as with any other general-education course. This can also be promoted through the youth' university/school emails, blackboards, and youth' orientation day and even on the notice boards both physically and the electronic. Secondly, the UAE universities/schools could include advertising the new "HEAL Public Campaign Course" on the nation's radio stations and television and other marketing mechanisms. This campaign could be in collaboration with the MOHESR, Knowledge and Human Development Agency (KHDA) and Abu Dhabi Education Council (ADEC) and others which would in turn send an indirect message on their involvement and concern with this public health issue among the youths of the country.

\section{Engagement of UAE communities}

The UAE youths could be asked to create videos and audios promoting healthier eating and active living with the goal of educating each other within the university and their respective peers, friends and families. Additionally, they could be asked to constructively critics each other's videos and audios before publicly posting onto the socialnetworking sites (SNS) such as the Face book accounts and LinkedIn wall pages. The youths could also be invited to participate in the getting into SHAPE program, where there would be an evaluation of the pre-, during and post- for the body-mass indexes, weight, body- fat analysis and maintaining a food dairy. There are two ways in which there can be the promotion of public relations within the target audience. The public relations is critical in terms of the identifying the significant public health issue-obesity, influencing the views of the public health issue, strengthening the advantageous opinions, transforming perhaps unbiased thoughts and ideas into optimistic ones, changing or neutralizing unreceptive feelings. One way could be through the public relations technique of promotional videos for the communication flow to occur and secondly through the SNS, such as Face book and LinkedIn and other websites.

The behaviour change on obesity is to promote modest lifestyle interventions among the youths within Dubai and the UAE at large, in additional to the promotional videos and audios' lessons for its prevention of obesity in the long run. The behaviors change would entail the maintenance of the suitable weight loss, lifestyle improvements, including continual healthier eating of diets, choosing and preference of healthier foods and beverages and continuing active living and exercises for 60-90minutes per day. ${ }^{22}$ The key benefits of such a behaviour change, would be, healthier lifestyles, longer healthier living, reduced communicable diseases, balanced between the calories consumed and calories expended, and increased work and studies productivity. ${ }^{23}$

Most of what we consume depends on our grocery shopping experience and our dining-out preference. Eating patterns have been attributed to our heritage and traditional background; however, obesity incidence has demanded that we keep nutritional topics in the forefront. ${ }^{24}$ One way to make this point is to understand the content nutritional food labels print and incorporate this information into sensible nutritional practices. According to Perez \& Edge, ${ }^{24}$ nutrition labelling refers to any identification of system that communicates the nutrient information of an individual food product. Nutritional food labelling is designed to inform consumers about the nutrient value in the kinds of foods consumed. Mindful of how many calories, fats, added sugars, carbohydrates, protein, and fiber a food or beverage contains can be part of the narrative to improve nutritional health. Every effort to address obesity here or aboard requires continual efforts to nudge the citizenry to change consumption behaviors using the knowledge that will not only inform but educate. ${ }^{24}$ Nutrient dense meals will improve nutritional health and combat obesity. ${ }^{25}$ The focus of nutritional health should continue to centre on population health.

There are potential stakeholders, community leaders, collaborative partners, or gate-keepers that may help to disseminate the message and encourage behavior change and their involvement. The World Health Organization (WHO) located in Dubai, the Ministry of Higher Education and Scientific Research (MOHESR), the universities/ schools can assist in the promotion and dissemination of the health eating and active living public health campaign via as a general education among the university youth. With the constant advertisement and media coverage by the MOHESR, this could also send a positive message for other universities to join in and collaborate in this venture towards promoting healthier eating habits and customs and increased of physical activities. Another way is that the MOHESR could enforce for this general education and/or workshops to be embedded and enforced as a compulsory/requirement course for all the accredited institutions by the Commission of Academic Accreditations from the MOHESR and KHDA licensed schools and institutions.

This general education course as mentioned earlier, the youth would be involved in some group work, therefore, another barrier or challenge would be to the categorization of the youth in suitable 
groups gender-wise and maybe language-wise. ${ }^{26}$ For instance, the female Emirati youth would need to group only into other female participants and vice versa for the males. Additionally, another way of addressing this would be to have female nurses, doctors and dietitians assigned to the female groups, and vice versa for the males of the target audience.

\section{Recommendations for reducing the prevalence of obesity}

The increasing prevalence and serious consequences of obesity have provoked public health campaigns for broad public health solutions that reach beyond clinic settings. ${ }^{27}$ Educational institutes are the ultimate surroundings for population-based intercessions to addressing obesity. ${ }^{27}$ In the implementation of the HEAL Public health campaign in the UAE has to be aligned with the main objective of promoting a healthier lifestyle through healthy eating and active living activities, in an effort to reduce obesity among the Arab youths within a University setting in Dubai, United Arab Emirates. There have many reports on the effectiveness of the health promotion and disease prevention programs with its target communities and audiences. ${ }^{28}$ The implementation process would include a number of public relations strategies such as the campus news media in order to provide an effective means of communicating the health information to the university. ${ }^{29,30}$

Another implementation method is conducting the self-assessment on the university youth using a School Health Index ${ }^{31}$ where the ratings of the eating habits, levels of physical activity could be recorded at the start, during and end of the 12-week HEAL campaign general education course. Additionally, prior to the implementation, staff trainings could be conducted in order to provide an opportunity to work together as a team and to share ideas with their equivalents. ${ }^{27}$ Another way of implementation is through the nutrition education where the university youth would be shown how to make better food choices and how to incorporate physical activities in order to influence a positive behavioral change. ${ }^{14,27}$ Another way for endorsing the HEAL public health campaign is through the development of a nutrition policy at the university where all the foods sold and served at the university campus could be in alignment with the nutritional guidelines and standards by the School Health Index. ${ }^{27}$ The Healthy Eating and Active Living (HEAL) public health message is one that could promote a healthy lifestyle through the incorporation of a balance diet based on the good food choices, the nutrients required for youths and how to maintain a healthy weight according to the World Health Organization standards..$^{32}$ This campaign in the writer's view can promote the change due to the nature of the message being realistic, allowing for a commitment, being consistent, flexible and creative. Our nutritional habits are formed around the meals prepared and the eateries we have come to enjoy over the years. How does one determine how much food is adequate for nutritional health? Is there a magic number to the amount of food one consumes daily? What should a balanced meal look like? Are these some questions ever asked?

Providing this information to consumers is an approach to encouraging nutritious mealtimes. A basic understanding will go a long way in fulfilling the requirement for healthy meals. Consulting nutrition labels can give the needed requirements for how much of a food item makes up a serving size. The food labels consider calories, fats, carbohydrates, protein, sugar, and fiber for each serving size. One must remember, however, that more than one serving can make up a food item. Consumers must take the time to read the nutrition food label to see the total amounts of the key components their dietary choice contains. This method of evaluation for nutritional health can offer lifelong learning benefits. Knowing how to read food labels can nudge and guide consumers away from obesity-related outcomes by actively participating in consulting nutritional information to improve nutritional health.

There are three ways in which the HEAL campaign be adopted through realistic and practical ways. One way is through the HEAL campaign sanctioning for the youths to forget about diet fads. In the end, diet is like fashion; it comes and goes without presenting an enduring solution. A typical food for thought question that would be asked is that, "Would I always eat this particular way, all my life?" Another way is to encourage the Arab youths to make a realistic commitment, in that they would be able to write down the imperative and significant motives for changing their respective eating habits and customs which can be shared as a verbal or written undertaking with their peers, colleagues, and family members and siblings. The third way is to promote the behavior change among the Arab youths through tactical means to do so. Many researchers have conclude that the for a behavior change to occur, it usually takes 21 days to do so. ${ }^{33}$ Therefore, as an example, within the campaign, promoting eating breakfast as part of a daily routine; the youth can be placed into groups and encourages planning their breakfast meals for the next 21 days of the healthy food items they love the most. It is important to be creative by thinking of all the possibilities in this example as well.

\section{Recommendations for the evaluation aspect}

The methods for the evaluation the effectiveness of the HEAL Campaign would be discussed as follows. One way is through weight management. The weight of each of the youth could be measured at the start of the campaign, then at the mid-way of the campaign and then the final week. Such an evaluative technique may provide information on the effectiveness of the program, regarding the progress and assessment of the youth' weight loss or gain in order to maintain a healthy weight during the campaign. Another way is to have open ended questions at the beginning of the campaign to have an idea of the health literacy levels and then pose the same questions at the end of the 12 weeks of the HEAL public health campaign and draw the conclusions of the effectiveness. Another way is through a debates competition at the twelfth week, where the youth would be asked to argue a real case study and properly assess the situation and proposed ways of helping to fix the dilemmas. Another way is to have games on reading food labels and identifying the good and bad items within the food items. Another way is to have the youth attain at least 2-3 peers at the beginning of the HEAL campaign (maybe from other classes) in order to promote the healthier eating habits and active living lifestyles. The youth could be assessed on how best they were able to convince their peers on healthy eating and active living.

There are two ways in which the HEAL public health campaign can promote social change. One way is through the promotion of daily healthier eating of balance diets which is a combination of just right amount of nutrients (carbohydrates, fats, proteins, minerals and vitamins) and portion controls. As a dietitian, an ideal healthy food plate is one that is colourful that are full many good nutrients. The second way is through the promotion of daily active living routines at the university campus which assists with the controlling of the blood lipid abnormalities, diabetes and obesity as well. Therefore, the exercise activities can be held at the university campus through 
walking, stair-climbing, jogging, running, swimming and sports. These two ways can promote social change among the youths at the university.

\section{Acknowledgements}

None.

\section{Conflict of interest}

The author declares no conflict of interest.

\section{References}

1. UAE Vision 2021. World-Class Healthcare Indicator. 2012.

2. Al-Haddad FH, Al-Nuaimi YI, Little BB, et al. The prevalence of obesity among school children in the United Arab Emirates. American Journal of Human Biology. 2000;12(4):498-502.

3. Al-Haddad FH, Little BB, Abdul-Ghafoor AM. Childhood obesity in United Arab Emirates schoolchildren: A national study. Annals of Human Biology. 2005;32(1):72-79.

4. Bagchi K. Nutrition in the eastern Mediterranean region of the World Health Organization. East Mediterr Health J. 2008;14 Suppl:107S-113S.

5. Malik M, Bakir A. Prevalence of overweight and obesity among children in the United Arab Emirates. Obes rev. 2007;8(1):15-20.

6. Zaal AB, Brebner J, Musaiger AO, et al. Anthropometric characteristics and obesity among adolescents in the United Arab Emirates. EMHJ 2011;17(5):382-386

7. Mahboub B, Safarainni B, Alhariri H, et al. Sleep breathing disorders in female population of Dubai, UAE. Health. 2013;5(12):2091-2096.

8. Ali HI, Ng SW, Zaghloul S, et al. High proportion of 6 to 18 -year-old children and adolescents in the United Arab Emirates are not meeting dietary recommendations. Nutrition Research. 2013;33(6):447-456.

9. Anekwe TD, Rahkovsky I. Economic cost and benefits of healthy eating. Current Obesity Reports. 2013;2:225-234.

10. Glanz K, Bishop DB. The role of behavioral science theory in development and implementation of public health interventions. Annu Rev Public Health. 2010;31:399-418.

11. United States Department of Health and Human Services (USDHHS). Theory at a glance: A guide for health promotion practice. 2nd ed. USA: National Institutes of Health; 2005.

12. Vanlandingham MJ, Suprasert S, Grandjean N, et al. Two views of risky sexual practices among northern Thai males: The health belief model and the theory of reasoned action. J Health Soc Behav. 1995;36(2):195-212.

13. Livingston IL, Saafir BD, Manuel R. Health knowledge among historically black college and university youth: an exploratory study. College Student Journal. 2012;46(3):581-588.

14. Brown SC, Geiselman PJ, Broussard T. Cardiovascular Risk in African American Women Attending Historically Black Colleges and Universities:The Role of Dietary Patterns and Food Preferences. $J$ Health Care Poor Underserved. 2010;21(4):1184-1193.
15. Hayes BD, Holliday RC, Wade BH, et al. A Comprehensive Examination of the Health Knowledge, Attitudes, and Behaviors of Youth Attending Historically Black Colleges and Universities. J Health Care Poor Underserved. 2009;20(2 Suppl):69-84.

16. Blackburn B. Japan earthquake and tsunami: Social media spreads news, raises relief funds. Japan: ABC World News; 2011.

17. Shen K, Khalifa M. Facebook usage among Arabic college youth: preliminary findings on gender differences. International Journal of eBusiness Management. 2010;4(1):53-65.

18. Solberg CA. Culture and industrial buyer behaviour: The Arab experience. $18^{\text {th }}$ IMP Conference, Africa; 2002.

19. Mourtada R, Salem F, Alshaer S. Citizen Engagement and Public Services in the Arab World: The Potential of Social Media. $6^{\text {th }}$ ed. United Arab Emirates: Arab Social Media Report Series, Mohammed Bin Rashid School of Government; 2014

20. United Nations. ILO Department of Statistics. USA: Face book User Penetration; 2014.

21. DiMaggio P, Hargittai E, Celeste C, et al. Digital inequality: From unequal access to differentiated use. In: Neckerman, editor. Social inequality. USA: Russell Sage Foundation; 2004. p. 355-400.

22. Hargittai, E, Shafer S. Differences in actual and perceived online skills: The role of gender. Social Science Quarterly. 2006;87(2):432-448.

23. WHO. Facts on Obesity. 2013.

24. Perez R, Edge MS. Global nutrition labelling: Moving toward standardization. Nutrition Policy. 2014;49(2):77-82.

25. Guthrie JF, Mancino L, Lin CJ. Nudging consumers toward better food choices: Policy approaches to changing food consumption behaviours. Psychology and Marketing. 2015;32(5):1-13.

26. Font JC, Hernandez-Quevedo C, McDonald JT, et al. Understanding healthy lifestyles: The role of choice and the environment. Applied Economic Perspectives and Policy. 2012;35(1):1-6.

27. Guion LA, Kent H, Diehl DC. Ethnic marketing: A strategy for marketing programs to diverse audiences. Florida: University of Florida; 2009.

28. Foster GD, Sherman S, Borradaile KE, et al. A policy-based school intervention to prevent overweight and obesity. Pediatrics. 2008;121(4):e794-e802.

29. Reger B, Wootan MG, Booth-Butterfield S, et al. $1 \%$ or less: a communitybased nutrition campaign. Public Health Reports. 1998;113(5):410-419.

30. Samuels SE. Project Lean-lessons learned from a national social marketing campaign. Public Health Reports. 1993;108(1):45-53.

31. Wallack L, Dorfman L, Jernigan D, et al. Media advocacy and public health. New York: Sage Publications; 1993.

32. Centers for Disease Control and Prevention. Welcome to the School Health Index: a self-assessment and planning guide. Atlanta, USA; 2012.

33. WHO. Global Strategy on Diet, Physical Activity and Health. 2013. 